\title{
Continuous infusion versus intermittent bolus injection of propofol during endoscopic retrograde cholangiopancreatography
}

Jae Gon Lee, Kyo-Sang Yoo, and Young Jae Byun

Department of Internal Medicine, Hanyang University Guri Hospital, Guri, Korea

Received: July 30, 2018

Revised : August 9, 2018

Accepted: November 15, 2018

\section{Correspondence to}

Kyo-Sang Yoo, M.D.

Department of Internal

Medicine, Hanyang University

Guri Hospital, 153 Gyeongchun-

ro, Guri 11923, Korea

Tel: +82-31-560-2229

Fax: +82-31-555-2998

E-mail: kyoo@hanyang.ac.kr
Background/Aims: It is unclear whether continuous infusion or intermittent bolus injection of propofol is better for achieving adequate sedation in endoscopic retrograde cholangiopancreatography (ERCP). We aimed to compare the efficacy and safety of continuous infusion and intermittent bolus injection of propofol during therapeutic ERCP.

Methods: In this prospective study, we randomly assigned 232 patients undergoing therapeutic ERCP to either continuous infusion (CI group, $\mathrm{n}=113$ ) or intermittent bolus injection (BI group, $\mathrm{n}=119$ ) of propofol. The primary outcome was the quality of sedation as assessed by the endoscopist. Other sedation-related parameters included sedation induction time, total dose of propofol, recovery time, involuntary patient movement, and adverse events.

Results: Overall satisfaction with sedation by the endoscopist and monitoring nurse were significantly higher in the CI group than the BI group (mean satisfaction score, 9.66 vs. 8.0 and 9.47 vs. 7.96, respectively, $p<0.01$ for both). However, patients in the CI group had a significantly longer sedation induction time (5.28 minutes vs. 4.34 minutes, $p<0.01$ ) and received a higher dose of propofol than patients in the BI group ( $4.22 \mathrm{mg} / \mathrm{kg}$ vs. $2.08 \mathrm{mg} / \mathrm{kg}, p<0.01)$. There was no significant difference in adverse events between the two groups.

Conclusions: Continuous infusion of propofol during therapeutic ERCP had the advantage over intermittent bolus injection of maintaining a constant level of sedation without increasing adverse events. However, it was associated with an increased total dose of propofol and prolonged sedation induction time.

Keywords: Propofol; Deep sedation; Endoscopic retrograde cholangiopancreatography

\section{INTRODUCTION}

Propofol is now widely used for sedation in gastrointestinal endoscopy, especially for therapeutic endoscopy procedures [1-3]. It has many advantages over benzodiazepines and/or opioids, including rapid onset of action, short half-life, fast recovery, and high levels of satisfac- tion for both patient and endoscopist $[2,4,5]$.

Propofol is generally administered by intermittent bolus injection with or without benzodiazepines and/or opioids [6]. Because of the rapid onset of action and short half-life of propofol, intermittent bolus injection may be associated with peaks and valleys of plasma propofol concentration. Peak levels can cause sedation-relat- 
ed adverse events such as hypoxemia and hypotension, while valleys can lead to rapid lightening of the sedative effect and subsequent involuntary patient movement [7]. Continuous propofol infusion via an infusion pump is an alternative method that could theoretically maintain more constant levels of sedation without fluctuations of plasma concentration, helping avoid involuntary patient movement and sedation-related adverse events [8].

A constant level of sedation is important, especially in complex and long-lasting therapeutic endoscopic procedures such as endoscopic retrograde cholangiopancreatography (ERCP). Involuntary patient movement under sedation can lead to procedure-related complications. In addition, the need for greater amounts of sedative to inhibit involuntary movement may increase the risk of sedation-related adverse events and prolong the procedure [5].

It is unclear whether continuous infusion or intermittent bolus injection of propofol is superior for achieving an adequate level of sedation during therapeutic ERCP. Only one study has compared continuous infusion and intermittent bolus injection during ERCP and endoscopic ultrasonography in terms of efficacy and safety, and its results were inconclusive [7]. Therefore, we designed a prospective, randomized study comparing the efficacy and safety of continuous infusion and intermittent bolus injection of propofol during therapeutic ERCP.

\section{METHODS}

\section{Study population}

All consecutive patients eligible for ERCP were enrolled between August 2012 and December 2013. We excluded patients who were under 19 years of age, pregnant, had known allergies to the drugs used or a history of sedation-related complications (i.e., severe paradoxical response, hypoxemia, bradycardia, and hypotension) or gastrectomy, were American Society of Anesthesiologist (ASA) class [9] $\geq \mathrm{IV}$, or were unable to provide informed consent. Patients were also excluded from the final analysis if the procedure was terminated early because of failure of bile duct cannulation due to anatomical abnormalities such as duodenal stenosis, previous gastric surgery, or periampullary anatomical variation.

\section{Study design and procedures}

This was a prospective, randomized study in a single tertiary referral center comparing the efficacy and safety of continuous infusion and intermittent bolus injection of propofol during therapeutic ERCP.

An independent physician randomized the patients to either the continuous infusion (CI) or intermittent bolus injection (BI) group at the point of sedation induction using a computer program. The allocation was concealed from the patients, endoscopist, and monitoring nurse, who only monitored the patients during the procedure. An independent physician and sedation nurse who did not participate in the survey were the only persons aware of the sedation method used for each patient. The independent physician determined the dosage of all sedatives taking into account age, body weight, and general condition of each patient, and the sedation nurse administered sedatives. To conceal the sedation procedure from the operator, an infusion pump was prepared for all procedures before randomization and was not removed, even if the patient was assigned to the BI group, and a screen was placed between the operator and sedation nurse.

In the CI group, the initial infusion rate of propofol was determined by the independent physician to be in the range of 9 to1 $2 \mathrm{mg} / \mathrm{kg} / \mathrm{hr}$. After induction of sedation, the infusion rate was lowered to the range of 2 to 5 $\mathrm{mg} / \mathrm{kg} / \mathrm{hr}$, then titrated by 1 to $2 \mathrm{mg} / \mathrm{kg} / \mathrm{hr}$ depending on patient sedation level [10,11].

In the BI group, sedation was initiated with an intravenous injection of a bolus of 1 to $2 \mathrm{mg}$ midazolam plus 20 to $30 \mathrm{mg}$ propofol. To maintain deep sedation, additional boluses of 10 to $20 \mathrm{mg}$ propofol were administered at intervals of at least 20 to 30 seconds [11]. The registered nurse who administered the propofol was certified in advanced cardiac life support and had completed a structured training program developed in our institution for administering propofol under the supervision of a physician. The endoscopist, independent physician, and monitoring nurse were also certified in advanced cardiac life support.

All patients fasted overnight before undergoing ERCP except in emergency cases and received $20 \mathrm{mg}$ hyoscine butylbromide and $25 \mathrm{mg}$ meperidine as premedications unless contraindicated. The procedure was performed with a standard duodenoscope (JF-240, TJF-24O, or TJF- 
260V, Olympus Optical, Tokyo, Japan) with the patient in a prone position. All endoscopic procedures were performed by one experienced endoscopist (K.S.Y.) who had performed more than 2,500 ERCP operations.

The study protocol was approved by the Institutional Review Board of Hanyang University Guri Hospital(IRB No. 2012-055). Written informed consent was obtained from all patients.

\section{Patient monitoring}

The patient's level of consciousness and vital signs were assessed just before the start of the procedure. Monitoring included pulse oximetry, 3-lead electrocardiography, automated non-invasive blood pressure and heart rate measurements. All patients received supplemental oxygen at a rate of $2 \mathrm{~L} / \mathrm{min}$, delivered by nasal cannula [6]. To assess the level of sedation, we used Modified Observer's Assessment of Alertness/Sedation (MOAA/S) scores. If the patient did not respond to mild prodding or shaking (MOAA/S score 1), or responded only after mild prodding or shaking (MOAA/S score 2), we determined that sedation was deep [12].

During the procedure, the patient's level of consciousness, vital signs including respiratory rate, oxygen-saturation, and involuntary movement were recorded every 5 minutes [6]. Equipment for emergency resuscitation was available at all times.

After completion of the procedure, the patient was monitored in the ERCP unit until complete recovery was achieved. Aldrete's scoring system, including activity, respiration, circulation, consciousness, and oxygen saturation, was used to determine the discharge. An Aldrete's score of 9 or more was considered appropriate for discharge [12].

\section{Study outcome}

Primary outcome was the quality of sedation as assessed by the endoscopist. The endoscopist and monitoring nurse completed a questionnaire using a $10-\mathrm{cm}$ visual analog scale (VAS), including overall satisfaction and difficulty in maintaining deep sedation during the procedure. In addition, all patients completed a questionnaire to evaluate overall satisfaction with the procedure and sedation once they had fully recovered from sedation.

Sedation induction time, total dose of propofol, recovery time, and frequency of involuntary patient move- ment were also recorded. Sedation induction time was defined as the time from first injection to deep sedation. Recovery time was defined as the time from completion of the procedure to complete recovery. All involuntary patient movement was recorded by the monitoring nurse. Any interruptions of the procedure of $>1$ minute due to severe involuntary movement were also recorded.

Sedation-related adverse events including hypoxemia $\left(\mathrm{SpO}_{2}<90 \%\right)$, hypotension (systolic blood pressure < $90 \mathrm{mmHg}$ ), and bradycardia (heart rate $<50$ beats/min) lasting more than 10 seconds were also evaluated. When hypoxemia occurred, we increased oxygen supply and performed airway management such as jaw thrust, bagmask ventilation, and endotracheal intubation, as appropriate.

\section{Statistical analysis}

All statistical analyses were performed with IBM SPSS Statistics version 20.0 (IBM Co., Armonk, NY, USA). The $t$ test and the chi-square test or Fisher's exact test were used to compare continuous and categorical data, respectively. A p value of $<0.05$ was considered statistically significant. Based on a previous report, we assumed that the mean \pm standard deviation satisfaction score of the endoscopist in the BI group would be $8 \pm 2$ [13-15]. Accordingly, to achieve $80 \%$ power to detect a difference of $10 \%$ ( $0.8 \mathrm{~cm}$ on a 10-cm VAS scale) with a two-sided test at an $\alpha$ value of 0.05 , a sample size of 99 patients per group was calculated. Considering a dropout rate of $10 \%, 218$ patients were needed.

\section{RESULTS}

\section{Patients}

A total of 255 patients were enrolled, of whom 15 were excluded initially due to severe comorbidity (ASA class $\geq$ IV) or previous history of gastrectomy. The 240 remaining patients were randomly assigned to either continuous infusion (CI group, $\mathrm{n}=117$ ) or intermittent bolus injection (BI group, $\mathrm{n}=123$ ) of propofol for deep sedation. Four patients with anatomical abnormalities in the CI group and three patients with anatomical abnormalities and one with current anticoagulant use in the BI group were excluded from the final analysis. A total of 232 patients, 113 in the CI group and 119 in the BI group, were 
finally analyzed (Fig. 1).

Baseline characteristics of the study population are listed in Table 1. There were no significant differences in age, sex, BMI, or indications for ERCP between the two groups. The mean age was 67.3 years, and the majority of patients underwent ERCP because of choledocholithiasis with or without acute cholangitis.

\section{Clinical outcomes}

Table 2 shows the results for sedation efficacy according to the two propofol injection methods. In terms of the quality of sedation, satisfaction of the endoscopist

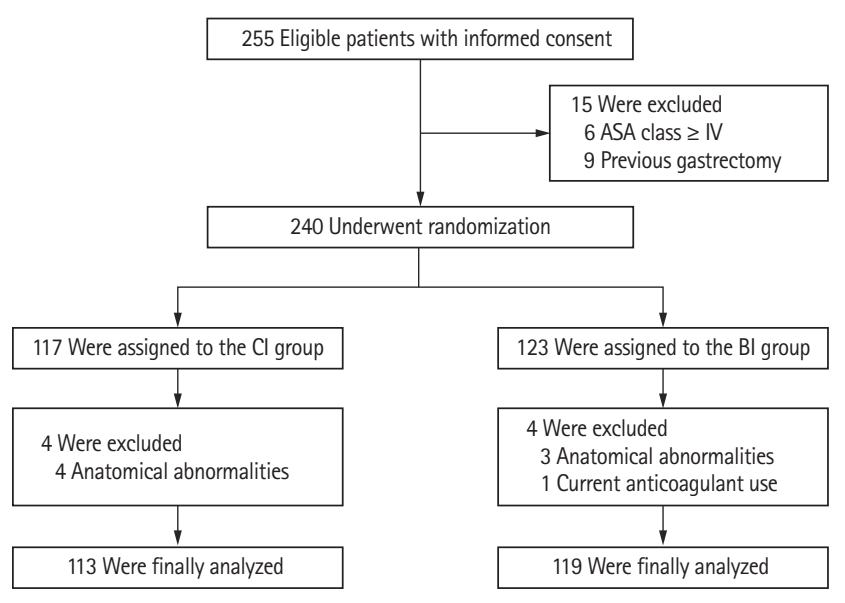

Figure 1. Flow diagram of the study. ASA, American Society of Anesthesiologist; CI, continuous infusion; BI, bolus injection. and monitoring nurse were significantly higher in the CI group: the mean satisfaction scores of the endoscopist and monitoring nurse were 9.66 and 9.47 in the CI group versus 8.0 and 7.96 in the BI group, respectively ( $p$ $<0.01$ for both). The mean scores for difficulty in maintaining deep sedation of the endoscopist and monitoring nurse were significantly lower in the CI group than in the BI group (1.11 vs. 2.54, $p<0.01$ and 1.54 vs. $2.66, p<$ 0.01 , respectively). Patient satisfaction with sedation was also higher in the CI group than in the BI group (mean satisfaction score, 9.64 vs. 9.07, $p<0.01$ ). Moreover, the frequency of involuntary movement was significantly lower in the CI group than in the BI group (4 events vs. 25 events, $p<0.01$ ), and no procedures were interrupted in the CI group compared to four interruptions in the BI group $(p=0.12)$.

However, the mean sedation induction time was significantly longer (5.28 minutes vs. 4.34 minutes, $p<0.01$ ) and the mean recovery time tended to be longer in the CI group than in the BI group (8.14 minutes vs. 7.04 minutes, $p=0.06$ ). Furthermore, the mean total dose of propofol per body weight was significantly higher in the CI group than in the BI group (4.22 mg/kg vs. $2.08 \mathrm{mg} /$ $\mathrm{kg}, p<0.01$ ).

\section{Adverse events}

Hypoxemia occurred in six of the 113 patients in the CI group, and four of the 119 patients in the BI group (5.3\%

Table 1. Baseline characteristics of the study population

\begin{tabular}{|c|c|c|c|}
\hline Characteristic & CI group $(n=113)$ & BI group $(n=119)$ & $p$ value \\
\hline Age, yr & $65.8 \pm 16.8$ & $68.9 \pm 14.6$ & 0.13 \\
\hline Sex & & & 0.69 \\
\hline Male & $56(49.6)$ & $55(46.2)$ & \\
\hline Female & $57(50.4)$ & $64(53.8)$ & \\
\hline $\mathrm{BMI}, \mathrm{kg} / \mathrm{m}^{2}$ & $23.1 \pm 3.4$ & $23.2 \pm 3.7$ & 0.83 \\
\hline Indication for ERCP & & & 0.70 \\
\hline Choledocholithiasis & $82(72.6)$ & $79(66.4)$ & \\
\hline Bile duct cancer & $16(14.2)$ & $20(16.8)$ & \\
\hline Biliary pancreatitis & $4(3 \cdot 5)$ & $3(2.5)$ & \\
\hline Pancreatic cancer & $6(5 \cdot 3)$ & $7(5 \cdot 9)$ & \\
\hline Other $^{\mathrm{a}}$ & $5(4.4)$ & $10(8.4)$ & \\
\hline
\end{tabular}

Values are presented as mean $\pm \mathrm{SD}$ or number $(\%)$.

CI, continuous infusion; BI, bolus injection; BMI, body mass index; ERCP, endoscopic retrograde cholangiopancreatography.

${ }^{a}$ Other includes intraductal papillary neoplasm, stricture of the common bile duct, and primary sclerosing cholangitis. 
Table 2. Parameters associated with sedation efficacy

\begin{tabular}{|c|c|c|c|}
\hline Variable & CI group $(n=113)$ & BI group $(n=119)$ & $p$ value \\
\hline \multicolumn{4}{|l|}{ Satisfaction of endoscopist } \\
\hline Overall satisfaction & $9.66 \pm 1.00$ & $8.0 \pm 1.68$ & $<0.01$ \\
\hline Difficulty score & $1.11 \pm 0.41$ & $2.54 \pm 1.73$ & $<0.01$ \\
\hline \multicolumn{4}{|l|}{ Satisfaction of monitoring nurse } \\
\hline Overall satisfaction & $9.47 \pm 1.48$ & $7.96 \pm 1.80$ & $<0.01$ \\
\hline Difficulty score & $1.54 \pm 1.02$ & $2.66 \pm 1.83$ & $<0.01$ \\
\hline \multicolumn{4}{|l|}{ Satisfaction of patient } \\
\hline Overall satisfaction & $9.64 \pm 0.74$ & $9.07 \pm 1.12$ & $<0.01$ \\
\hline Sedation induction time, min & $5.28 \pm 2.07$ & $4.34 \pm 2.44$ & $<0.01$ \\
\hline Involuntary patient movement & $4(3.5)$ & $25(21.0)$ & $<0.01$ \\
\hline Interruptions of procedure & o & $4(3 \cdot 4)$ & 0.12 \\
\hline Procedure time, $\min$ & $22.14 \pm 12.64$ & $20.72 \pm 10.87$ & 0.36 \\
\hline Recovery time, min & $8.14 \pm 4.83$ & $7.04 \pm 4.07$ & 0.06 \\
\hline Total dose of propofol, mg/kg & $4.22 \pm 1.73$ & $2.08 \pm 1.35$ & $<0.01$ \\
\hline Total dose of midazolam, mg & - & $1.65 \pm 0.51$ & - \\
\hline
\end{tabular}

Values are presented as mean $\pm \mathrm{SD}$ or number (\%).

$\mathrm{CI}$, continuous infusion; BI, bolus injection.

vs. $3.4 \%, p=0.53)$. No patients required endotracheal intubation, but two patients in each group required bagmask ventilation (1.8\% vs. $1.7 \%, p=1.00$ ). When $\mathrm{SpO}_{2}$ decreased to less than $90 \%$, the oxygen supply was increased to $5 \mathrm{~L} / \mathrm{min}$ through the nasal cannula. If $\mathrm{SpO}_{2}$ was decreasing or did not increase to $\geq 90 \%$ within 1 minute despite the oxygen increase, the procedure was immediately stopped and the endoscope was removed. An oropharyngeal airway was placed, propofol infusion was discontinued in the CI group, and intravenous flumazenil was administered in the BI group. Bag-mask ventilation was performed with an oxygen supply of $15 \mathrm{~L} /$ min. All four patients that required bag-mask ventilation recovered within 2 minutes. The procedure was resumed with a reduced propofol dose of $50 \%$ to $70 \%$ of the last dose, and all procedures were completed successfully.

Systolic hypotension, defined as systolic blood pressure $<90 \mathrm{mmHg}$, occurred in one patient in the CI group and no patients in the BI group $(p=0.49)$. In the patient with hypotension, the infusion rate of propofol was lowered by 1 to $2 \mathrm{mg} / \mathrm{kg} / \mathrm{hr}$ and intravenous saline was administered. Blood pressure recovered within 2 minutes without interruption of the procedure. No bradycardia occurred in either group. Overall, there were no significant differences in adverse events between the two groups (Table 3 ).

\section{DISCUSSION}

Propofol-based sedation is now widely used in gastrointestinal endoscopy. Therapeutic endoscopic procedures such as ERCP, however, require deep sedation without respiratory compromise [12], and meticulous dose adjustment is necessary when administering propofol. Propofol-based sedation, including balanced propofol sedation and propofol monosedation, is well-established, as are methods of administration, including intermittent bolus injection and continuous infusion; however, no single method has been found to be superior to the others [16].

In the present work, we observed that the overall satisfaction of the endoscopist and monitoring nurse with sedation were both significantly higher when propofol was administered by continuous infusion than when it was administered by intermittent bolus injection, and there was no significant difference in adverse events between the two procedures. 
Table 3. Adverse events

\begin{tabular}{|c|c|c|c|}
\hline Variable & CI group $(n=113)$ & BI group $(n=119)$ & $p$ value \\
\hline Hypoxemia & $6(5 \cdot 3)$ & $4(3.4)$ & 0.53 \\
\hline Bag-mask ventilation & $2(1.8)$ & $2(1.7)$ & 1.00 \\
\hline Endotracheal intubation & o & 0 & - \\
\hline Hypotension & $1(0.9)$ & 0 & 0.49 \\
\hline
\end{tabular}

Values are presented as number (\%).

$\mathrm{CI}$, continuous infusion; BI, bolus injection.

The overall satisfaction of the endoscopist, monitoring nurse, and patients with sedation was high in the CI group; the endoscopist was not disturbed by involuntary patient movement during the procedure, and the monitoring nurse found it easy to maintain the level of sedation. Indeed, involuntary patient movement was significantly less frequent in the CI group than the BI group. Theoretically, continuous infusion should be more effective in maintaining a constant level of sedation than intermittent bolus injection because it can maintain constant plasma propofol concentrations [8]. However, a previous study comparing the efficacy and safety of continuous infusion versus intermittent bolus injection of propofol during ERCP found no significant differences in patient cooperation, total propofol dose, or adverse events between the methods. Because the authors of that study administered $3 \mathrm{mg}$ of midazolam, a higher dose than in the present study, the fluctuation of any sedation effects with intermittent propofol injection may have been reduced, leading to inconclusive results [7]. In the current study, however, it is likely that constant levels of sedation were achieved with constant plasma propofol concentrations in the CI group, although we did not measure plasma concentrations. In contrast, in the BI group, involuntary patient movement was frequent and 4 procedures had to be interrupted as a result. Involuntary patient movement probably reflects the difficulty in predicting when to inject successive propofol boluses, leading to peaks and valleys of plasma propofol concentration. These results suggest that continuous infusion is superior to intermittent bolus injection for maintaining a constant level of sedation.

Although continuous infusion showed better results in terms of the quality of sedation than intermittent bolus injection, we found that it was associated with a higher total dose of propofol and longer sedation induc- tion time, in contrast to a previous study that found no significant difference in total propofol dose between the methods [7]. In the current study, a low dose of midazolam was administered concomitantly with propofol only in the BI group according to the standard of balanced propofol sedation, which may have led to a lower total dose of propofol and shorter sedation induction time in the BI group, because of the propofol-sparing effect of midazolam [7]. In addition, although the difference was not statistically significant, continuous infusion was also associated with prolonged recovery time, in agreement with the previous study [7]. This outcome may be explained by the fact that propofol infusion was discontinued concurrently with the end of the procedure in the CI group, whereas the last dose was administered several minutes before the end of the procedure in the BI group.

Despite the high dose of propofol administered in the CI group, there was no significant difference in adverse events between the two groups. Delivering a higher dose of propofol can be dangerous because there is no reversal agent, and sedation-related adverse events such as hypoxemia and hypotension are associated with the total dose and infusion rate of propofol [17-19]. However, the incidence of hypoxemia did not increase significantly in the CI group in association with the higher administered dose of propofol. Presumably, the total dose of propofol was higher in the CI group because midazolam was not administered concomitantly, and the low incidence of hypoxemia may have been due to the preserved pharyngeal function associated with continuous infusion, as reported in a previous study [19].

This study has some limitations. First, we assessed the quality of sedation using the arbitrary and subjective indicator of satisfaction of the endoscopist and nurse, because there were no proper objective indicators to assess 
the quality of sedation. To complement this approach, we also evaluated patient satisfaction. Second, we administered midazolam concomitantly with propofol in the $\mathrm{BI}$ group, and propofol monosedation was performed in the CI group. Therefore, direct comparison of the total dose of propofol between the two groups may be inappropriate because midazolam may have had a propofol-sparing effect. Third, the study was conducted in a single institution, making it difficult to generalize the results. Total doses of propofol and midazolam were lower than in previous studies $[20,21]$. Furthermore, since the incidence of adverse events was lower than in previous studies [22,23], we detected no statistically significant differences in the frequency of adverse events between the two groups. A larger scale, multinational and multicenter study is needed to overcome these limitations.

Despite these limitations, this study offers a better understanding of propofol administration methods during ERCP. In conclusion, continuous infusion of propofol during therapeutic ERCP is superior for maintaining a constant level of sedation and endoscopist satisfaction than intermittent bolus injection without an increased risk of adverse events, but is associated with an increased total dose of propofol and prolonged sedation induction time.

\section{KEY MESSAGE}

1. During therapeutic endoscopic retrograde cholangiopancreatography, continuous infusion of propofol was superior for maintaining a constant level of sedation compared to intermittent bolus injection.

2. Continuous infusion of propofol was associated with an increased total dose of propofol and prolonged sedation induction time, but not with an increased risk of adverse events.

\section{Conflict of interest}

No potential conflict of interest relevant to this article was reported.

\section{Acknowledgments}

This work was supported by the research fund of Hanyang University (HY-2012).

\section{REFERENCES}

1. Cohen LB, Wecsler JS, Gaetano JN, et al. Endoscopic sedation in the United States: results from a nationwide survey. Am J Gastroenterol 2006;101:967-974.

2. Jung M, Hofmann C, Kiesslich R, Brackertz A. Improved sedation in diagnostic and therapeutic ERCP: propofol is an alternative to midazolam. Endoscopy 2000;32:233-238.

3. Riphaus A, Stergiou N, Wehrmann T. Sedation with propofol for routine ERCP in high-risk octogenarians: a randomized, controlled study. Am J Gastroenterol 2005;100:1957-1963.

4. McQuaid KR, Laine L. A systematic review and meta-analysis of randomized, controlled trials of moderate sedation for routine endoscopic procedures. Gastrointest Endosc 2008;67:910-923.

5. Dumonceau JM, Riphaus A, Aparicio JR, et al. European Society of Gastrointestinal Endoscopy, European Society of Gastroenterology and Endoscopy Nurses and Associates, and the European Society of Anaesthesiology Guideline: non-anesthesiologist administration of propofol for GI endoscopy. Endoscopy 2010;42:960-974.

6. ASGE Standards of Practice Committee, Early DS, Lightdale JR, et al. Guidelines for sedation and anesthesia in GI endoscopy. Gastrointest Endosc 2018;87:327-337.

7. Riphaus A, Geist C, Schrader K, Martchenko K, Wehrmann T. Intermittent manually controlled versus continuous infusion of propofol for deep sedation during interventional endoscopy: a prospective randomized trial. Scand J Gastroenterol 2012;47:1078-1085.

8. Shafer A, Doze VA, Shafer SL, White PF. Pharmacokinetics and pharmacodynamics of propofol infusions during general anesthesia. Anesthesiology 1988;69:348-356.

9. Doyle DJ, Garmon EH. American Society of Anesthesiologists Classification (ASA Class). Treasure Island (FL): StatPearls Publishing, 2018 [cited 2019 Sep 15]. Available from: http://www.ncbi.nlm.nih.gov/books/NBK441940.

10. Choi GJ, Kang H, Baek CW, Jung YH, Lee JJ. Comparison of bolus versus continuous infusion of propofol for procedural sedation: a meta-analysis. Curr Med Res Opin 2017;33:1935-1943.

11. Moon SH. Sedation regimens for gastrointestinal endoscopy. Clin Endosc 2014;47:135-140.

12. American Association for Study of Liver Diseases; American College of Gastroenterology; American Gastroenterological Association Institute, et al. Multisociety sedation 
curriculum for gastrointestinal endoscopy. Gastrointest Endosc 2012;76:e1-e25.

13. Lee TH, Lee CK, Park SH, et al. Balanced propofol sedation versus propofol monosedation in therapeutic pancreaticobiliary endoscopic procedures. Dig Dis Sci 2012;57:2113-2121.

14. Agostoni M, Fanti L, Arcidiacono PG, et al. Midazolam and pethidine versus propofol and fentanyl patient controlled sedation/analgesia for upper gastrointestinal tract ultrasound endoscopy: a prospective randomized controlled trial. Dig Liver Dis 2007;39:1024-1029.

15. Shin S, Park CH, Kim HJ, Park SH, Lee SK, Yoo YC. Patient satisfaction after endoscopic submucosal dissection under propofol-based sedation: a small premedication makes all the difference. Surg Endosc 2017;31:2636-2644.

16. Cohen LB, Delegge MH, Aisenberg J, et al. AGA institute review of endoscopic sedation. Gastroenterology 2007;133:675-701.

17. Qadeer MA, Vargo JJ, Khandwala F, Lopez R, Zuccaro G. Propofol versus traditional sedative agents for gastrointestinal endoscopy: a meta-analysis. Clin Gastroenterol Hepatol 2005;3:1049-1056.

18. Seifert H, Schmitt TH, Gultekin T, Caspary WF, Wehrmann T. Sedation with propofol plus midazolam versus propofol alone for interventional endoscopic procedures: a prospective, randomized study. Aliment Pharmacol Ther 2000;14:1207-1214.

19. Kiriyama S, Naitoh H, Fukuchi M, et al. Evaluation of pharyngeal function between no bolus and bolus propofol induced sedation for advanced upper endoscopy. Diagn Ther Endosc 2014;2014:248097.

20. Fanti L, Agostoni M, Casati A, et al. Target-controlled propofol infusion during monitored anesthesia in patients undergoing ERCP. Gastrointest Endosc 2004;60:361366.

21. Wehrmann T, Kokabpick S, Lembcke B, Caspary WF, Seifert H. Efficacy and safety of intravenous propofol sedation during routine ERCP: a prospective, controlled study. Gastrointest Endosc 1999;49:677-683.

22. Angsuwatcharakon P, Rerknimitr R, Ridtitid W, et al. Cocktail sedation containing Propofol versus conventional sedation for ERCP: a prospective, randomized controlled study. BMC Anesthesiol 2012;12:20.

23. Klare P, Reiter J, Meining A, et al. Capnographic monitoring of midazolam and propofol sedation during ERCP: a randomized controlled study (EndoBreath Study). Endoscopy 2016;48:42-50. 